\title{
Interactive comment on
}

\section{"Competitionalterspredicted forest carbon cycle} responses to nitrogen availability and elevated $\mathrm{CO}_{2}$ : simulationsusing an explicitly competitive, game-theoreticvegetation demographic mode" by Ensheng Weng et al.

\section{Benjamin Stocker (Referee)}

b.stocker@creaf.uab.cat

Received and published: 12 March 2019

This paper describes a model and its prediction for competitively optimal allocation (ratio of root to foliage surface area) and how it varies with a range of nitrogen levels and two $\mathrm{CO} 2$ levels. The same (or a similar version of the same? See point 12 below) model has been described and applied previously to investigate optimal phenological strategies in Weng et al. (2016, GCB, doi: 10.1111/gcb.13542) and a carbon only 
version was presented in Weng et al. (2015, BG, doi:10.5194/bg-12-2655-2015).

The present manuscript addresses allocation as a single variable parameter, although multiple traits affect plant functioning in the face of $\mathrm{N}$ availability and $\mathrm{CO} 2$ concentrations. However, the focus on allocation is justified, in my opinion, for two reasons: First, allocation warrants particular attention as it is a key process that is known to be responsive to changes in above and belowground resource availabilities and affects the carbon cycling in ecosystems when an allocation shifts occur between long-lived wood and short-lived foliage or fine roots. Second, most vegetation models simulate allocation either based on fixed parameters, or based on empirical relationships. However, as pointed out in the manuscript (l. 528), predicting allocation from first principles is key to realistically and robustly simulating vegetation changes in response to multiple changing environmental factors. The present model embodies a promising way forward to resolve this challenge, determining a competitively optimal allocation strategy, based on height-structured competition for light in the form of a Vegetation Demographics Modelling approach (cohort-based, not average individual-based as is common in Dynamic Vegetation Models). In this respect, the present model takes a pioneering path, that only less than a handful (or even less?) other models can follow.

I see two main weaknesses of the present study. First, predictions are not tested against observational data. What data needs to be used as a test (see comment 10)? However, I don't expect much additional insight from a comparison to observational data at this stage, and consider the theoretical predictions to deserve to be presented as "naked predictions". However, for a paper that deals with just the theoretical side of the problem, some aspects may warrant additional attention (additional figures for results results and extended discussion) in the manuscript (see comments 6, 8, 10, 11). Second, the modelling approach with regards to the excess-C-sapwood allocation (see I.245) raised some questions for me. Is it compatible with our understanding for what controls sapwood area (or what determines the Huber value, defined as the ratio of sapwood area:leaf area)? I worry that this model formulation may cause unrealis-

Printer-friendly version

Discussion paper 
tic model behaviour in some instances. Anyways, the conclusions need to be drawn carefully with regards to this aspect (see comment 5 below).

In the "SPECIFIC POINTS" described below, I am suggesting some modifications in the description of the model and to improve readability, and some changes in the presentation to distill the most relevant points and most interesting insights from this research. GENERAL POINTS may warrant particular attention. The paper is generally written very well and the presentation of results is clear and clean. If these points can be addressed satisfactorily, I may recommend a revised version of this manuscript for publication in Biogeosciences.

\section{GENERAL POINTS}

1. Dynamic adaptation (adaptive plastic responses) of allocation occurs also within species. The present model embodies the assumption that species follow a fixed allocation strategy, and changes in allocation are induced by replacement of species with different allocation strategies. Please add a discussion for the assumption of fixed within-species allocation may affect results.

2. A competitively optimal strategy is determined for stationary boundary conditions. Does this inhibit such a modelling approach to be applicable for global change simulations (transiently changing boundary conditions)? See also comment 11.

3. Allocation and the plant-level $\mathrm{C}$ and $\mathrm{N}$ budgets, assume fixed tissue $\mathrm{C}: \mathrm{N}$ ratios and flexible allocation to sapwood to make $\mathrm{C}$ and $\mathrm{N}$ budgets work. This does not correspond to the known controls on sapwood area and may induce unwanted model behaviour. However, it is difficult to think understand to what degree this affects the results and conclusions. See also comment 5.

4. Total $\mathrm{N}$ that is circulating in the system is manipulated for the different simulations, with zero $\mathrm{N}$ loss and inputs. This sounds like a rather pragmatic than realistic solution. In reality, losses are never zero, and $\mathrm{N}$ levels are manipulated in experiments by

Printer-friendly version

Discussion paper
Interactive comment

C3 
fertilisation. Why is it not implemented like this? Would you expect any systematic differences between your implementation and one with non-zero losses and manipulated inputs?

5. The abstract mentions "opposite fractional allocation to fine roots and wood" in competitive and fixed-allocation runs. Relatively more allocation to fine roots at high $\mathrm{N}$ levels in fixed allocation runs sounds like a result that runs counter to the common understanding of the controls on fine root allocation (e.g. Poorter et al., $2012 \mathrm{New}$ Phytologist, doi: 10.1111/j.1469-8137.2011.03952.x), and appears confusing in view of the fact that the model is based on a fixed root:leaf area ratio. I suspect that the increase in relative root allocation at high $\mathrm{N}$ levels in the fixed-allocation run is a result of the excess-C-sapwood allocation in this model. See also my comment 3 . If this is indeed the case, I would recommend not to present results in the abstract that are contingent on this, arguably unrealistic, model behaviour. I guess the authors don't want to challenge the common understanding of fine root allocation controls with their results. It would suffice to point out that in the competition runs, relative fine root allocation decreases with increasing $\mathrm{N}$ levels; and present this in the light of the common modelling approach whereby the root:leaf area ratios (and effective relative allocation ratios) are fixed.

6. Presentation of results for a fixed $\varphi$ : In my understanding, the essence of this research is to predict how stand-level relative allocation changes in response to $\mathrm{N}$ availability and $\mathrm{CO} 2$. The approach to predict it is to derive a competitively optimal allocation strategy at the individual-level. In view of the main aim (essence) of this research, I would expect as a (first) figure something that shows competitively optimal $\varphi$ for each N/CO2 level, derived from the competition runs.

7. A question: Does each point in Fig. 3 show the wood/root/foliage NPP (fraction) for the competitively optimal $\varphi$ at the respective $\mathrm{N}$ and $\mathrm{CO} 2$ levels (for the competition runs)? Comparing this to values from fixed-allocation runs with a pre-specified $\varphi$ is maybe not the most interesting. This leads to the next point. . .

Printer-friendly version

Discussion paper 
8. [see Addendum to this comment] In view of my comment 1 (dynamic adaptation of allocation also within species), readers may want to know if an optimality criterion that is defined for some instantaneous individual-level metric (like foliage NPP, or height growth, etc.) leads to the (qualitatively) same predictions as an optimality criterion that is funded in an evolutionarily stable strategy (ESS, like the one applied here). It might be interesting to evaluate the fixed-allocation runs to determine which $\varphi$ maximises some optimality criterion (like foliage NPP, or height growth, etc.). A (first) figure could then compare this individual-level derived optimal $\varphi$ to the ESS-derived optimal $\varphi$. Furthermore, points in Fig. 3 for the fixed-allocation runs could then be taken to represent the $\varphi$ level that maximises the optimality criterion for each N/CO2 level respectively. This would also enable a direct comparison of the the two optimality approaches (ESS vs. instantaneous). I understand if this suggestion is beyond the scope of this paper, or not feasible. Anyways, I would be interested to learn more about such a comparison. Addendum: I see now (upon reading the Discussion, I. 425), that this point is addressed by Supplementary figures S1 and S2. Maybe this is too much of my own personal interest, but I think to generally enhance this point in the presentation of the results (possibly with additional/modified figures as suggested above) would improve the manuscript.

9. A description of how the competitively optimal allocation is determined (description of the algorithm), would be helpful. May be added before current Section 2.2.

10. The differences in predictions based on different allocation schemes are interesting, but the missing comparison to observational data prevents conclusions to be drawn about which is more realistic or leads to better model performance. The question is: What is the key observation that can be used to test predictions? And of course: What is the key prediction that authors want to test? In my view, it is a viable option to remain with theoretical predictions, not actually using data from observations. However, this may require an extended discussion in the light of generally observed patterns with additional references to the literature. The challenge remains that the overproliferation

Printer-friendly version

Discussion paper
Interactive comment 
in root growth predicted by the competitively-optimal allocation scheme may not directly be testable. How much is over-proliferation in reality? What would be a suitable observation to evaluate this prediction?

11. Regarding implications for Earth system modelling: From reading this manuscript, it's not entirely clear whether the approach for determining the competitively-optimal allocation strategy is applicable for typical Earth system simulations, where boundary conditions change transiently. In my understanding, the approach chosen here determines a system steady state, formed by a monospecific stand with a certain allocation strategy, that cannot be invaded by any species with a different allocation strategy. As explained in the manuscript, this requires the model to be run into steady state for 1200 simulation years. How would this be implemented for a typical Earth system simulation setup? I think it would be very informative to complement Section 4.3 with a discussion on this point.

12. The description of how the present model version differs from model versions used in Weng et al., 2016, 2015, could be made clearer.

\section{SPECIFIC POINTS}

1.96: Should be a gap in 'trait (s)'?

Eq. 2: To solve the model, ds/dt has to be set to zero, I guess. Shouldn't this be reflected in Eq. 2? Or how exactly is the competitively optimal strategy determined?

I.148: Apart from variations in across-species allocation patterns (e.g., oak species tending to invest more into roots), there are also clear patterns in within species and within-individuals (flexible adaptation) variations in allocation when subjected to shifts in resource availability. In my understanding, such fast allocation responses are not captured by the modelling approach here. This should be clarified. Connects to Comment 1 above.

I.159: The simulation experiments are described in the abstract and intro to be done

Printer-friendly version

Discussion paper 
along a "nitrogen availability gradient". How did you manipulate N? This is described at a later stage, but could already be made clear here.

Section 2.1: A separate paragraph on how $\mathrm{CO} 2$ assimilation is simulated, would be helpful.

Eq. 3: To be consistent with Fig. 1, I would suggest to use the symbol X_FR as the pool size (or C_FR in this case), distinguish community- and individual-level variables for example using a bar over the variable for the community-level, and use a separate letter for the parameter 'Root_0' (e.g., K_FR).

Eq. 4: Should be clarified that this is the community-level total root biomass (if I am correct). Clarification is needed to understand Eq. 4.

Eq. 5: $\mathrm{D}$ is not defined. Diameter?

I. 215: Add bracket: “... targets for leaf, fine root, and sapwood cross-sectional area $\left(L^{*} \_k, F R^{*} k\right.$, and $\left.A^{*} \_S W\right)$ " here for a better overview of the description.

Eq. 5/6: How is $D$ incremented? The way the system is described now, the tree doesn't grow in $\mathrm{D}$ or am I missing something?

I. 238: Can you add f_1 and f_2 to the description in this sentence? E.g., "capped by a larger fraction of NSC (f_1)"?

I. 241: Are Eq. 7 and its parameters $f \_1$ and f_2 identical throughout the year? The description here suggests that something is different during leaf flush versus the period of "normal growth". Or maybe l'm just misunderstanding it the way it's formulated now.

I. 246: Units of 0.15 ? g C?

I. 254: Since sapwood production requires $\mathrm{N}$ as well (although relatively less than production of other tissues), and "excess C" sounds like this is the amount of NSC left, after NSN is used up (hence zero), I would assume that some iteration is necessary to perfectly match the use of NSC and NSN in the allocation procedure. How is this

Printer-friendly version

Discussion paper 
solved? Either more detail should be given here, or the description should be modified to avoid misunderstanding.

I. 260: I would welcome a summarising sentence on the mechanisms determining C:N stoichiometry. The connection between sapwood allocation and the NSC:NSN budget implies that a plant that acquires relatively little $\mathrm{N}$ in comparison to assimilated $\mathrm{C}$ (in other words: an "N-limited tree") would produce relatively more sapwood. Does this mean that Eq. 6 (the A_SW sub-equation) is "over"-satisfied? What are the implications of this in the model? Does it affect the relationship between height growth vs. crown area expansion?

Section 2.1: A description of how the competitively optimal allocation is determined (description of the algorithm) would be helpful. May be added before current Section 2.2 .

I. 292: "Full factorial" suggests that all combinations of treatment factors are applied to force the runs. But here, this is a mix of a treatment factor ( $\mathrm{N}$ levels) and model parameter (phi). I suggest to rephrase this.

Table 1: If $\mathrm{i}=(114.5, \ldots, 552) \mathrm{g} \mathrm{N} \mathrm{m}-2$, then $4.5-0.5^{*} \mathrm{i}$ is a negative number. Is this correct? Maybe $\mathrm{N}$ levels in units of $\mathrm{kg} \mathrm{N} \mathrm{m-2}$ are used here?

I. 362-364: The decrease in fractional allocation to fine roots with elevated $\mathrm{CO} 2$ at high $\mathrm{N}$ levels is surprising. May it be a result of the excess- $\mathrm{C}$ sapwood allocation approach implemented in this model? May warrant a brief discussion of this aspect.

I. 386-388: In my reading, this is a main result and should be shown in a separate figure, shown at the very beginning of the results section.

I. 418-419: See my comment 5.

Printer-friendly version

I.425: See my comment 8 . This is an interesting point, but is dealt with rather briefly here. How is "maximising growth rate" implemented exactly? NPP? NPP of a specific pool? "Allocating very little" is vague. The crucial aspect is that for a given $\mathrm{N}$ level and 
uptake half-saturation constant, the plants allocate much less to fine roots in the bestperforming (by what measure?) fixed-allocation run than in the competitively optimalallocation run.

I. 430-433: Sentence is hard to follow. Is the height at transition into the canopy (reaching critical height) increasing or decreasing with increasing $N$ ?

I. 493 ("succession"): Discussing competitively optimal strategy shifts during succession confused me here. I understood, that the the competitively optimal strategy is determined for a *steady-state*, and (based on my understanding from reading previous papers of this group of authors) an ESS is determined from competition upon invasion into a mono-specific stand. But now I realise that the algorithm for determining competitive optimality has never been described in the present paper. A gap that shold be filled (see also comments above).

I. 569: I had a great laugh when I read the short description of that repository on github ("BiomeESS: for simulating multiple plant forms, on-going, unpublished, with ridiculous processes and many bugs.") Maybe the author wants to change that upon publication of this manuscript (and if necessary resolve some known bugs). If not, I appreciate the honesty.

Interactive comment on Biogeosciences Discuss., https://doi.org/10.5194/bg-2019-55, 2019. 